\title{
Polarimetric Radar Interferometry for Improved Mine Detection and Surface Clutter Rejection
}

\author{
Lluís Sagués, Student Member, IEEE, Juan Manuel Lopez-Sanchez, Member, IEEE, \\ Joaquim Fortuny, Associate Member, IEEE, Xavier Fabregas, Member, IEEE, Antoni Broquetas, Member, IEEE, \\ and Alois J. Sieber, Fellow, IEEE
}

\begin{abstract}
A recently developed technique, polarimetric radar interferometry, is applied to tackle the problem of the detection of buried objects embedded in surface clutter. An experiment with a fully polarimetric radar in an anechoic chamber has been carried out using different frequency bands and baselines. The processed results show the ability of this technique to detect buried plastic mines and to measure their depth. This technique enables the detection of plastic mines even if their backscatter response is much lower than that of the surface clutter.
\end{abstract}

Index Terms-Interferometry, radar polarimetry, subsurface sensing, synthetic aperture radar (SAR).

\section{INTRODUCTION}

$\mathbf{S}$ YNTHETIC aperture radar (SAR) is a remote sensing technique that has been recently used to detect and identify buried mines. Airborne SAR systems have been found to be an excellent tool for survey of mine fields at a large scale [1]. Several vehicle-mounted forward looking SAR systems have also been developed in order to detect and identify every single mine. However, the detection performance of these systems is only acceptable when looking for large metallic mines or UXO. Buried plastic mines are nearly invisible to the radar and can not be detected due to the low dielectric contrast between the mine and the surrounding soil [1]. Moreover, the weak signal returned from the mine is normally obscured by the surface clutter, making its detection even more difficult. In this context, polarimetric SAR interferometry is a new technique that can be applied to enhance the detectability of the mine embedded in the surface clutter.

It is well known that conventional SAR interferometry uses relative phase information from two radar images acquired from

Manuscript received June 8, 2000; revised October 31, 2000. This work was supported by the Spanish Commission for Science and Technology (CICYT) under TIC99-1050-C03-01. This work was carried out in the framework of the European Commission TMR Network on Radar Polarimetry under ERB-FMRX-CT98-0211.

L. Sagués and X. Fabregas are with the Departament de Teoria del Senyal Comunicacions, Universitat Politecnica de Catalunya, 08034 Barcelona, Spain (e-mail: sagues@ voltor.upc.es).

J. M. Lopez-Sanchez is with the Departamento de Física, Ingeneria Sistemas y Teoría de la Señal (DFISTS), Universidad de Alicante, 03080 Alicante, Spain (e-mail: juanma@disc.ua.es).

J. Fortuny and A. J. Sieber are with the Space Applications Institute, Joint Research Centre of the European Commission, 21020 Ispra, Italy (e-mail Joaquim.Fortuny@jrc.it).

A. Broquetas is with the Institut de Geomatica, 08038 Barcelona, Spain (e-mail: toni@tsc.upc.es).

Publisher Item Identifier S 0196-2892(01)04846-X. different viewing angles to estimate the height of ground scatterers. On the other hand, radar polarimetry enables the extraction of target information from the measurements of the polarization state of the scattered waves, thus making it possible to decompose the scene into different scattering behaviors. Therefore, by combining both polarimetry and interferometry one can retrieve the height of different scattering mechanisms present in the same resolution cell, even though one scattering process is much weaker than the other [2], [3]. This important feature is based on the exploitation of the orthogonality between scattering mechanisms, that is utilized by the combination of polarimetry and interferometry.

This paper presents a polarimetric interferometry technique that is capable of separating the phase center corresponding to the mine from that related to the clutter and estimating their relative height difference or depth. Several multifrequency experiments have been carried out in the anechoic chamber of the European Microwave Signature Laboratory (EMSL) at JRC Ispra, Italy [4]. Fully coherent polarimetric data were collected in the frequency range 2-6 GHz. The measurement scene consisted of four US Army mine simulants and a metallic cylinder buried in dry sand below a thin layer of gravel (see details in the body of the paper). All results are shown and discussed in this paper. The potential application of polarimetric interferometry to subsurface detection was originally stated in [3], however this paper constitutes the first demonstration of this technique with experimental data.

The text is organized as follows. The formulation and methodology of the proposed technique are described in Section II. Section III shows the experiments performed in this study and the analysis of the results derived by applying this method. A summary of the results is discussed in Section IV, where some possible lines of future research about this technique are outlined as well.

\section{Methodology}

This section firstly presents a brief review of SAR interferometry in application to a buried mine scenario. Then, the method for combining both polarimetry and interferometry is described, and the technique for extracting the height or depth of the target under observation is explained.

\section{A. SAR Interferometry}

The geometry of a generic interferometric imaging system is illustrated in Fig. 1. Two complex SAR images, $s_{1}$ and $s_{2}$, are obtained from slightly different viewing angles. The antennas 


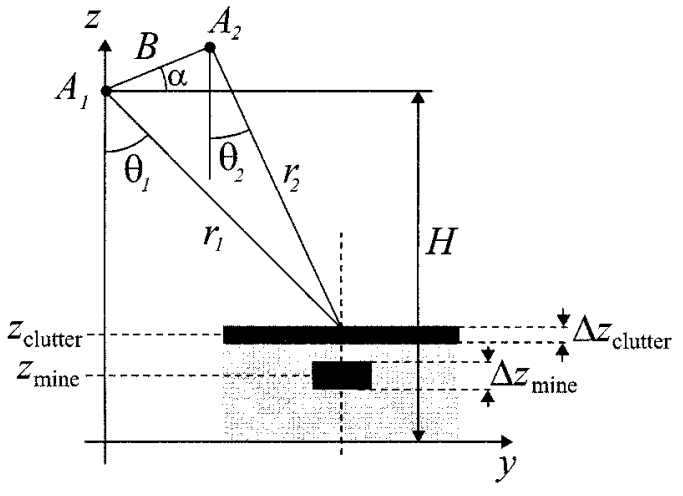

Fig. 1. Generic interferometric SAR system operating in a buried mine scenario.

are located at two different positions $A_{1}$ and $A_{2}$, illuminating the same patch on the surface at two incidence angles $\theta_{1}$ and $\theta_{2}$, respectively. The distance between the two antenna positions is known as baseline $B$, whereas the distances from each antenna position to the center of the resolution cell are $r_{1}$ and $r_{2}$.

The interferogram is generated by multiplying one image by the complex conjugate of the other. Thus, the phase of the interferogram $\phi_{\text {int }}$ corresponds to the phase difference between both SAR images

$$
\phi_{\text {int }}=\operatorname{angle}\left(s_{1} s_{2}^{*}\right)=\phi_{1}-\phi_{2}=\frac{4 \pi}{\lambda}\left(r_{2}-r_{1}\right)=\frac{4 \pi}{\lambda} \Delta r .
$$

Since the phase of each SAR image is related to the distance between the antennas and the ground, a relationship between this travel path difference and the local height of each ground scatterer can be derived

$$
\begin{aligned}
\sin (\alpha-\theta) & =\frac{(r+\Delta r)^{2}-r^{2}-B^{2}}{2 r B} \\
h & =H-r \cos \theta
\end{aligned}
$$

whereby we can generate an elevation map of the scene under observation. Here, $r$ and $\theta$ are the mean values of $r_{1}$ and $r_{2}$, and $\theta_{1}$ and $\theta_{2}$, respectively. Hence, an additional height dimension is introduced in the reflectivity images. The quality of the generated elevation map depends on the quality of the interferometric phase. The parameter used to evaluate the phase quality is the so-called interferometric coherence, $\gamma$, which can be interpreted as a useful tool for measuring the resemblance or correlation between both SAR images

$$
\gamma=\frac{\left\langle s_{1} s_{2}^{*}\right\rangle}{\sqrt{\left\langle\left|s_{1}\right|^{2}\right\rangle\left\langle\left|s_{2}\right|^{2}\right\rangle}} .
$$

If the coherence equals to zero, the scene is completely uncorrelated and then the interferogram is noisy and not related to the topography. At the other extreme, a coherence close to one corresponds to a noise-free interferogram from which a high quality elevation map can be generated.

In a real scenario (see Fig. 1), the buried mine is embedded in the surface clutter. The separation of the different scattering centers inside the resolution cell (i.e., the surface clutter and the mine) by using conventional SAR interferometry is not possible. Therefore, a key step would be to separate the scattering mechanism of the mine from that associated with the surface clutter in order to get their relative heights. Polarimetry becomes useful at this point: any scattering mechanism can be identified as a particular polarization state of the wave. Hence, the separation of these scattering mechanisms is based on selecting those polarization states in both images that maximize the interferometric coherence. As a result, the interferometric phase associated with them exhibits a better quality and the height estimation is more accurate.

\section{B. Coherence Optimization by Polarimetry}

The optimization algorithm employed in this paper is known as polarization subspace method (PSM). The PSM is based on finding the local maxima of the copolar or crosspolar coherence functions that have been calculated from the measurements of all polarization states of the scattered wave. The PSM was already described in [5], but is also briefly outlined here for the sake of completeness.

When fully coherent polarimetric data are collected, the information associated with each pixel of the SAR image is defined by the following scattering vector (assuming a backscattering configuration)

$$
\underline{k}=\left[S_{h h}, \sqrt{2} S_{h v}, S_{v v}\right]^{T}
$$

where $S_{m n}$ is the complex scattering coefficient for $n$ transmitted and $m$ received polarization expressed in the orthogonal $(h, v)$ basis. In the case of interferometric measurements, the polarimetric information will be contained in two different scattering vectors, $\underline{k}^{1}$ and $\underline{k}^{2}$, for image 1 and image 2 , respectively, leading to the following Hermitian matrices:

$$
\begin{aligned}
{\left[C^{i j}\right] } & =\left\langle\underline{k}^{i} \underline{k}^{j * T}\right\rangle \\
& =\left[\begin{array}{ccc}
\left\langle S_{h h}^{i} S_{h h}^{j *}\right\rangle & \sqrt{2}\left\langle S_{h h}^{i} S_{h v}^{j *}\right\rangle & \left\langle S_{h h}^{i} S_{v v}^{j *}\right\rangle \\
\sqrt{2}\left\langle S_{h v}^{i} S_{h h}^{j *}\right\rangle & 2\left\langle S_{h v}^{i} S_{h v}^{j *}\right\rangle & \sqrt{2}\left\langle S_{h v}^{i} S_{v v}^{j *}\right\rangle \\
\left\langle S_{v v}^{i} S_{h h}^{j *}\right\rangle & \sqrt{2}\left\langle S_{v v}^{i} S_{h v}^{j *}\right\rangle & \left\langle S_{v v}^{i} S_{v v}^{j *}\right\rangle
\end{array}\right]
\end{aligned}
$$

where $i=1,2$ and $j=1,2$ refers to each SAR image used to form the interferogram. Thus, $\left[C^{11}\right]$ and $\left[C^{22}\right]$ are the two covariance matrices associated with the separate images, whereas $\left[C^{12}\right]$ is a $3 \times 3$ complex matrix which contains the interferometric information between polarimetric channels. Otherwise, instead of using a linear polarization basis to describe the vector fields, one can use any other orthogonal elliptic basis. The coordinates transformation of a scattering vector from the linear basis $(h, v)$ to another orthonormal elliptic basis $(x, y)$ can be accomplished by applying a unitary matrix transformation $\left[U_{3}\right]_{(\tau, \phi)}$ to the polarization vector [5]

$$
\underline{k}^{\prime}=\left[S_{x x}, \sqrt{2} S_{x y}, S_{y y}\right]^{T}=\left[U_{3}\right]_{(\tau, \phi)} \underline{k}
$$

where $\tau$ and $\phi$ are the ellipticity and inclination angles that define any polarization state. Physically, this transformation can be interpreted as a change of the selected scattering mechanisms in both images. After applying this polarization basis change, we can express the covariance matrices defined in (6) in any different polarization state $(\tau, \phi)$

$$
\left[C^{i j}\right]_{(\tau, \phi)}^{\prime}=\left\langle\left[U_{3}\right] \underline{k}^{i} \underline{k}^{j * T}\left[U_{3}\right]^{* T}\right\rangle=\left\langle\left[U_{3}\right]\left[C^{i j}\right]\left[U_{3}\right]^{* T}\right\rangle .
$$


The application of these polarimetric basis transformation enables the formation of interferograms between all possible elliptical polarization states and polarimetric combinations between both SAR images. In this way, the interferometric coherence between every polarimetric combination will be given by

$$
\begin{aligned}
\left|[\Gamma]_{(\tau, \phi)}\right| & =\left[\frac{\left|c_{m n}^{12^{\prime}}\right|}{\sqrt{c_{m n}^{11^{\prime}} c_{m n}^{22^{\prime}}}}\right]_{1 \leq m \leq 3,1 \leq n \leq 3} \\
& =\left[\begin{array}{lll}
\gamma_{x x-x x} & \gamma_{x x-x y} & \gamma_{x x-y y} \\
\gamma_{x y-x x} & \gamma_{x y-x y} & \gamma_{x y-y y} \\
\gamma_{y y-x x} & \gamma_{y y-x y} & \gamma_{y y-y y}
\end{array}\right]
\end{aligned}
$$

being $c_{m n}^{i j^{\prime}}$ the elements of the covariance matrices defined in (8). The optimum interferogram will be generated by selecting that combination of polarization states $(\tau, \phi)$ which maximizes the interferometric coherence $\gamma$. Once the interferometric phase is calculated, it can be converted to absolute height by using the conventional interferometric phase-to-height equations described in (3).

We can further simplify this approach by using some a priori knowledge about the values in $[\Gamma]_{(\tau, \phi)}$. The first assumption is that, in cases where there is no temporal decorrelation and the baseline is short, the highest coherence will be given by the same polarization state in both images, and therefore the elements of the diagonal of the $[\Gamma]$ matrix will be much higher than the rest. Moreover, the definition of the geometrical angles implies the following transformation symmetry

$$
\gamma_{x x-x x}(\tau, \phi)=\gamma_{y y-y y}(-\tau, \phi+\pi / 2)
$$

and, consequently, if the assumption is valid, we must calculate only the first and the second elements of the diagonal of $[\Gamma]$ and select the maximum one

$$
\gamma_{o p t}=\max \left\{\gamma_{x x-x x}(\tau, \phi), \gamma_{x y-x y}(\tau, \phi)\right\}
$$

This technique provides a graphical representation which can be very useful to derive the height structure of the target. By plotting the copolar and crosspolar coherence functions as a function of $(\tau, \phi)$, it is possible to identify the existence of independent scattering mechanisms inside the same resolution cell. In the case of a single dominant scattering behavior, both coherence functions generally present only one absolute maximum. However, in those cases where the target exhibits a multilayer vertical structure, as shown in Fig. 1, it is possible to recognize various local coherence maxima. These optimum polarization states will be related to different independent scattering mechanisms that lead to the locally best height estimation. In the case of buried mine detection, it is expected to find two coherence maxima: the mine and the surface clutter (or air-ground interface). After generating the two interferograms corresponding to these optimum polarization states, we can extract the height difference between them. The resulting height difference map will exhibit some peaks at those positions where the mines are buried, whereas it will remain close to zero at those zones of the image where only a natural surface scattering process can be found.
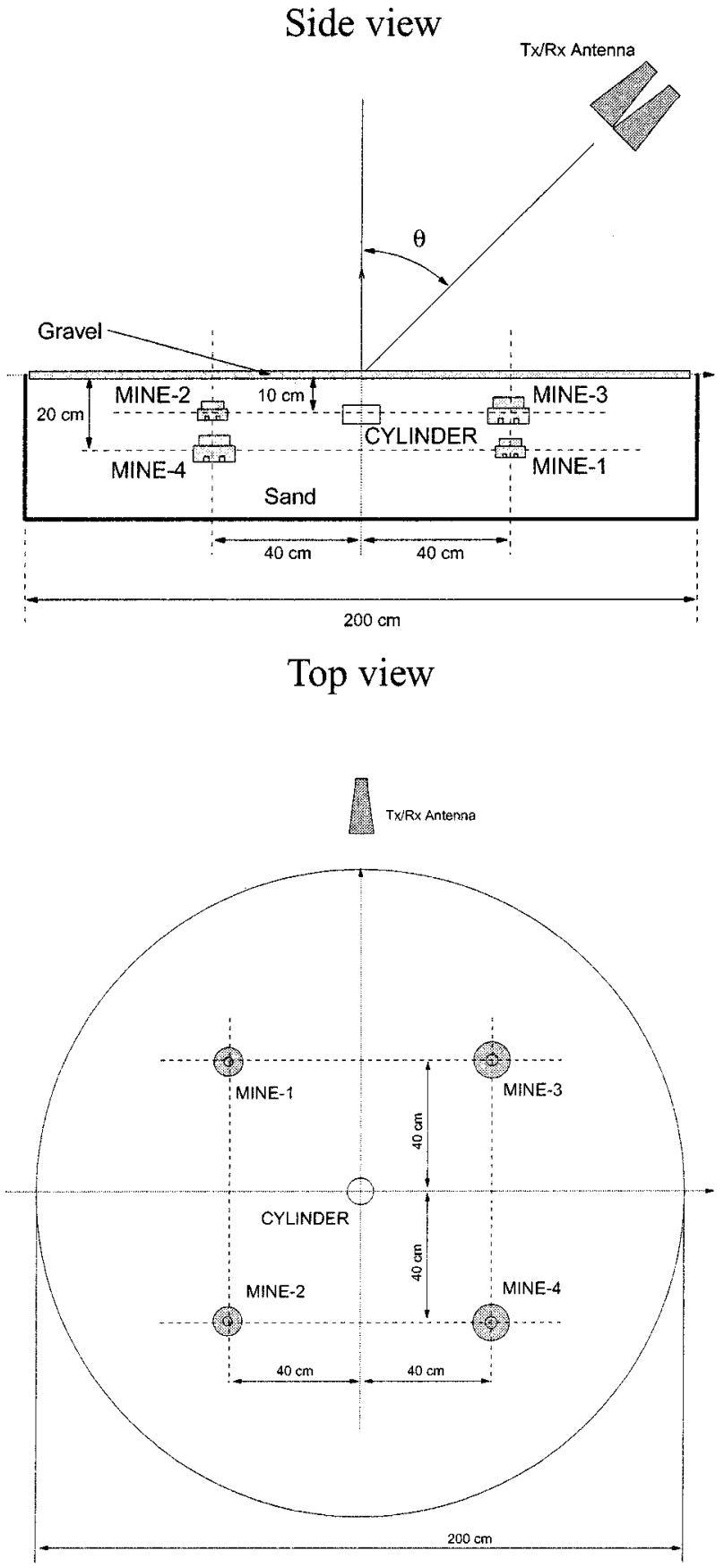

Fig. 2. Experimental setup: side and top view.

\section{EXPERIMENTAL RESULTS AND DISCUSSION}

A number of multifrequency experiments were carried out in the anechoic chamber of the European Microwave Signature Laboratory (EMSL) at JRC Ispra, Italy. The experimental setup is detailed in Fig. 2. Four plastic mines and a metallic cylinder were buried in dry sand under a layer of gravel $2 \mathrm{~cm}$ thick. The mines used were simulants gently provided by the U.S. Army. The primary filler of these simulants was RTV-3110 silicone rubber, which dielectric permittivity is approximately 3.0. The measured dielectric permittivity of the dry sand was about 2.5. Such a low dielectric contrast will make the detectability of the mine extremely difficult. Mine 1 and mine 4 were buried at 

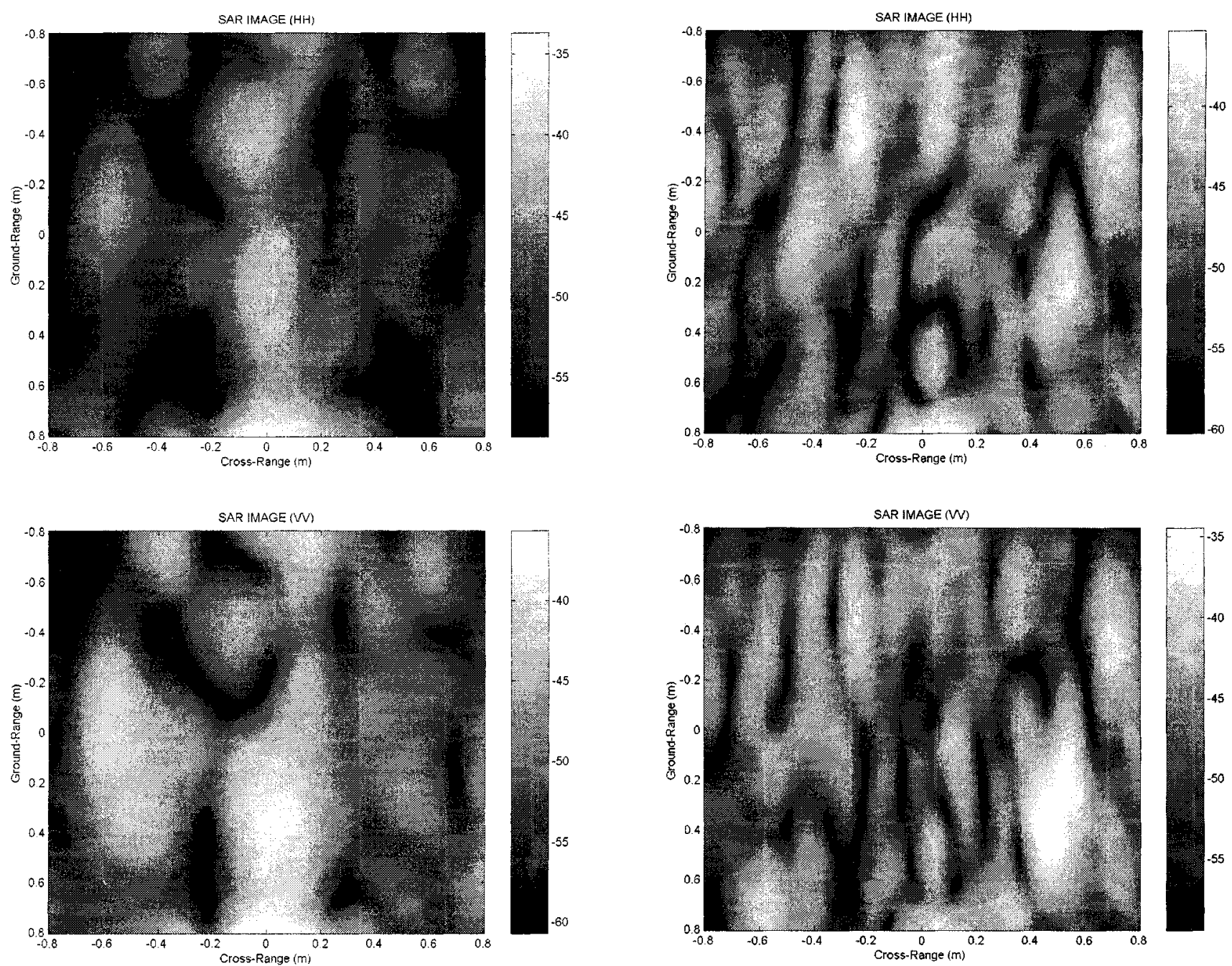

Fig. 3. HH (top) and VV (bottom) SAR amplitude images. Frequency $=2-3$ $\mathrm{GHz}$.

Fig. 4. HH (top) and VV (bottom) SAR amplitude images. Frequency $=4-5$ GHz.

$20 \mathrm{~cm}$ depth under the surface, whereas the other two mines were buried at $10 \mathrm{~cm}$ depth. The separation between mines was $80 \mathrm{~cm}$ and their size was different: the diameter of mines 1 and 2 was $20 \mathrm{~cm}$, whereas the diameter of mines 3 and 4 was $25 \mathrm{~cm}$. The metallic cylinder (with a height of $10 \mathrm{~cm}$ and a diameter of $20 \mathrm{~cm}$ ) was buried in the middle of the four mines at $10 \mathrm{~cm}$ depth. Fully coherent polarimetric data were collected for different baselines, covering the frequency range from 2 to $6 \mathrm{GHz}$. The center incidence angle was $45^{\circ}$, and the distance between the antennas and the target center was about $10 \mathrm{~m}$. The aperture was synthesized by moving the target with the use of a linear positioner. Note that the measurement is performed in a quasi-monostatic configuration. More details about the mechanization of the EMSL experiments can be found in [5]. In all results presented in the text, the baseline is expressed as the difference between both incidence angles: $B=\Delta \theta=\theta_{1}-\theta_{2}$.

Figs. 3 and 4 show the reconstructed SAR images using $\mathrm{HH}$ and VV polarization for the frequency ranges $2-3 \mathrm{GHz}$ and 4-5 GHz, respectively. The size of the resolution cell is about $20 \mathrm{~cm}$ in both dimensions. The metallic cylinder is easily identified in the scene at 2-3 GHz. In these reflectivity maps, the cylinder is located about $36 \mathrm{~cm}$ beyond the center of the scene

because the image was focused on a horizontal plane at the surface level. This displacement can be predicted by studying the refraction on an ideal air-sand interface and projecting onto the normal of the incidence direction. However, the four plastic mines can not be distinguished using the amplitude reflectivity image because the strong surface clutter completely masks the mine backscattering response. This is also the case for all buried targets (cylinder and mines) at 4-5 GHz. Note that the mines can not be identified in any image generated in this experiment, all with frequencies above $2 \mathrm{GHz}$. Therefore, an alternative approach must be employed to discriminate their response from the gravel backscattering.

The application of the proposed polarimetric interferometry technique is illustrated as follows. Fig. 5 depicts the obtained coherence function for all $(\tau, \phi)$ polarization states when they are computed from a $60 \times 60 \mathrm{~cm}$ window centered at the mine 3 location. We can notice that the copolar coherence function $\gamma_{x x-x x}(\tau, \phi)$ exhibits two local maxima. The two local maxima appear at $\left(\tau_{1}=42^{\circ}, \phi_{1}=10^{\circ}\right)$ and $\left(\tau_{2}=-22^{\circ}, \phi_{2}=44^{\circ}\right)$. Once both maxima are identified, we can proceed to calculate two interferograms: one with each polarization state. Finally, both interferometric phases are converted to heights. The abso- 

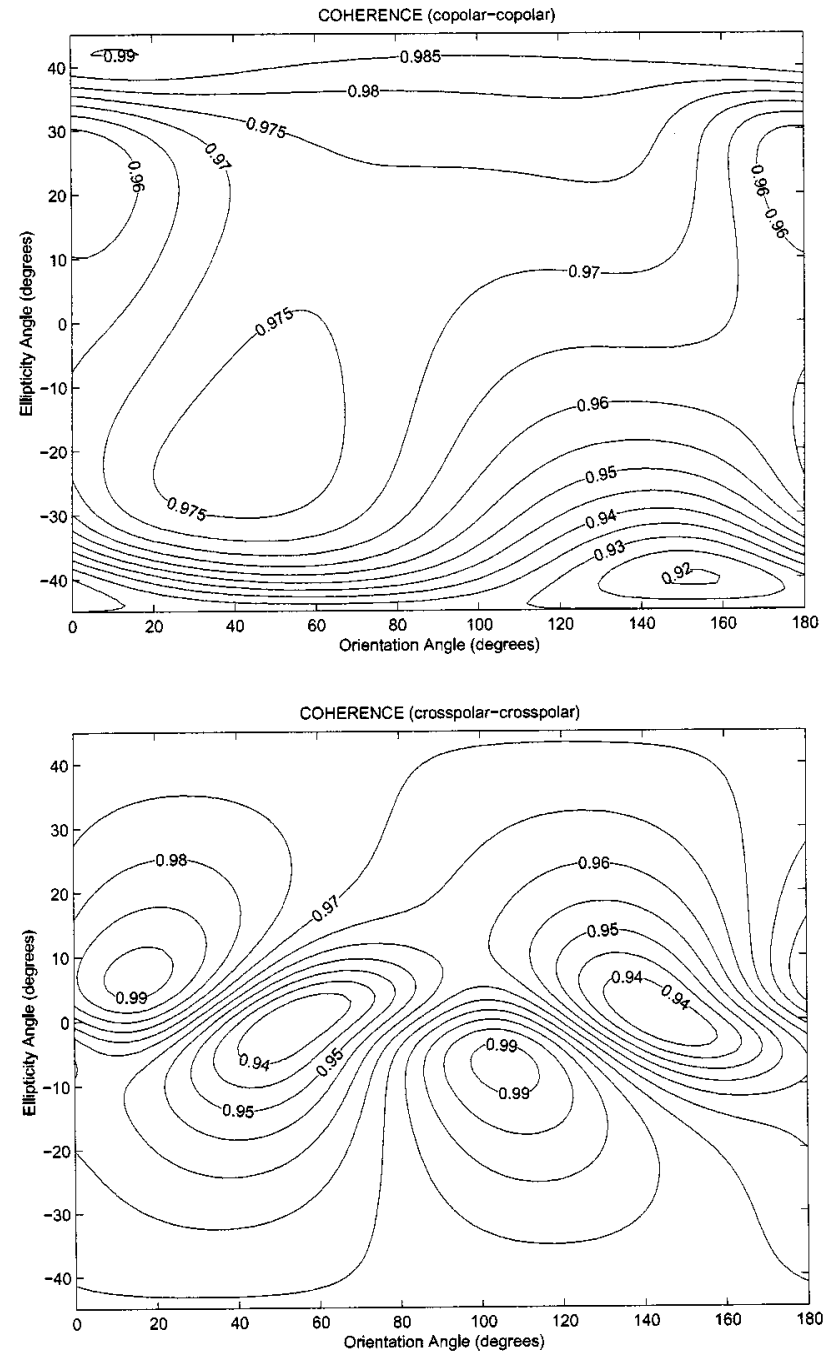

Fig. 5. Coherence as a function of polarization (copolar and crosspolar combinations) for the position of mine 3 . Frequency $=2-3 \mathrm{GHz} . B=0.5^{\circ}$

lute height associated with the first state of polarization is 0.7 $\mathrm{cm}$, which is very close to the surface reference height, whereas the height derived from the second one is $-8.54 \mathrm{~cm}$. Therefore, we can state that the first corresponds to the gravel scattering while the second is related to the buried mine. The height difference between both polarization states is $9.24 \mathrm{~cm}$, which is very close to the actual depth of mine $3(10 \mathrm{~cm})$. It is important to note that this depth estimate has been obtained by applying a multi-look procedure (spatial averaging of $N=9$ resolution cells) in order to reduce its standard deviation. When observing the crosspolar coherence function $\gamma_{x y-x y}(\tau, \phi)$, only a single maximum is found (it appears twice in this plot due to a known symmetry [5]). If this polarization state is employed in the interferogram generation, the estimated height is $-8.5 \mathrm{~cm}$. This height is in turn very close to the mine depth already provided by the copolar coherence, so we can assume that it is redundant. Consequently, the rest of results in this paper are computed by employing only the copolar coherence function.

The available $S / N$ in the crosspolar measurements is also an important topic to be discussed at this point. It is known that ideally any body of revolution (as cylinder and mines) does not

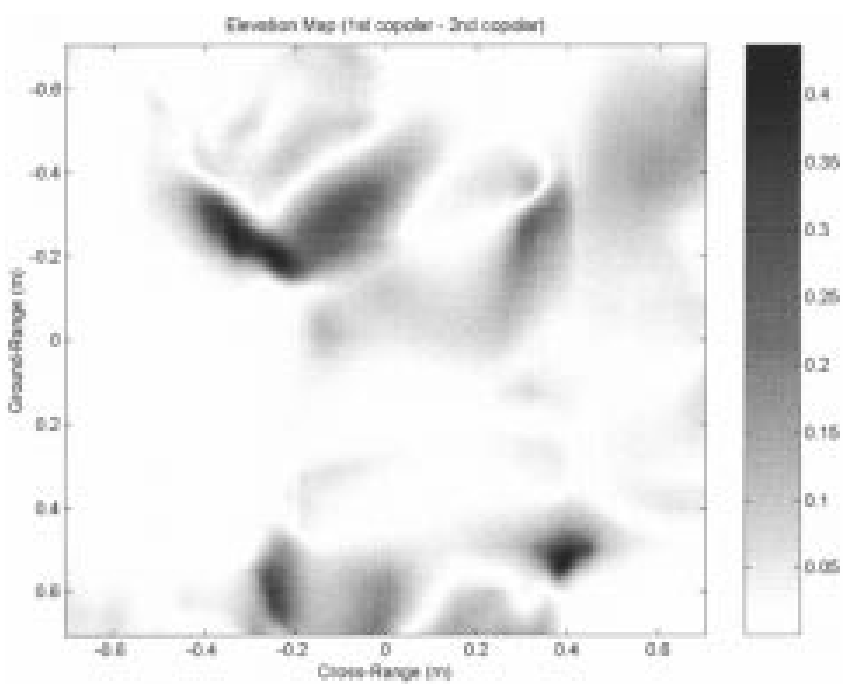

Fig. 6. Map derived from the height difference between the two optimum copolar polarizations at $2-3 \mathrm{GHz} . B=0.5^{\circ}$.

return any crosspolar signal, and only when tilted it has a significant backscattering amplitude. In the previous result there is a response strong enough for the crosspolar coherence to be different from the crosspolar coherence of the gravel alone. This can be demonstrated by comparing the coherence range of Fig. 5 and that shown in Fig. 4 of [5], which are 0.94-0.99 and $0.75-0.94$, respectively. This coherence increase is not due to the setup, but to the presence of the mine. We can justify this by making use of the following expression of the coherence [6]:

$$
\gamma=1-\frac{\Delta \theta}{\tan \theta} \frac{f_{o}}{W}
$$

where $f_{o}$ is the center frequency and $W$ is the bandwidth. With this expression the coherence values for both cases are: 0.978 and 0.956 , respectively.

The proposed procedure can be applied to the entire surface by sliding the averaging window on the resulting complex interferograms. In any case, it is not possible to consider a large number of spatial looks during the averaging process because they introduce a loss of spatial resolution. Fig. 6 shows the spatial map of the elevation (or depth) obtained by calculating the height difference between those polarization states that maximize the copolar coherence function, in the frequency range 2-3 GHz. The resulting height difference is large at the positions where the mines were buried, but it remains close to zero outside those spots. Therefore, all four buried mines can be clearly detected. Nevertheless, we note that the metallic cylinder is not identified in this map. In first instance the reason is that the ground attenuation at this frequency is low (or, equivalently, the penetration is high), and the resulting cylinder scattering amplitude is too high compared to the surface. Consequently, the copolar function, calculated at the cylinder zone, exhibits only a single maximum that corresponds to the cylinder. In fact, we saw in the images of Fig. 3 that the cylinder was clearly detected without the use of interferometry.

The previous argument is valid for scalar interferometry, but when dealing with polarimetric interferometry a more subtle process can also be involved. The performance of this technique 
depends on the orthogonality of the scattering mechanisms. In principle, if the buried target and surface clutter are orthogonal then detection will still be possible, even for large scattering amplitude differences. This backscattering difference, which could be quantified as a signal-to-clutter ratio, should remain within a realistic range but the boundaries of this interval are still subject of an ongoing research. On the other hand, if the scattering mechanisms are similar, then polarimetry will play a minor role anyway.

The same scheme has been applied to the frequency range 4-5 GHz. Fig. 7 illustrates the copolar coherence functions versus polarization state for three locations in the scene which correspond to a zone without buried objects, the cylinder, and mine 3 , respectively. At this frequency range, the coherence functions obtained from cylinder and mine positions present two maxima, whereas the gravel plot exhibits a unique maximum. The area without buried objects was expected to produce a single maximum, because the only scattering element is the gravel. In fact, the resulting maximum is very broad so we can deduce that the interferogram is practically independent of polarization. In the case of the cylinder, both gravel and cylinder provide coherence maxima because at the current frequency the gravel is not masked by the cylinder (in contrast with the previous result at 2-3 GHz). The zone of mine 3 produces two maxima but, unfortunately, their corresponding heights are very close together and close to the gravel level. We can conclude that the response from the mine is completely obscured by the clutter at this frequency range, even for this detection scheme. Hence, after calculating the height difference between the two optimum states of polarization over the whole scene, we obtain the result shown in Fig. 8. Note that in this case the cylinder is well detected whereas the mines can not be distinguished.

Another key point must be emphasized here. In this work we have employed the PSM in the coherence maximization. The PSM is a subspace method and hence searches for the coherence maximum in a subspace of possibilities. A coherence optimizer that searches in the full space was presented in [2]. Results obtained with this global method may clarify this discussion, but they are not available yet. In relation with this point, it is possible that the global optimizer could find a coherence maximum (for instance maximizing the mine return at the same time as minimizing the surface return) that lies in a space outside of the copolar response and hence will not be found with the PSM. This is a likely explanation for not detecting the cylinder in Fig. 6, since the coherence maxima of cylinder and surface may be close in the copolar subspace. In conclusion, the detectability is also affected by the optimization method.

In addition, it is known that plastic mines show a stronger polarization dependence than metallic objects since the former exhibit internal wave reflections. These are particularly important in the range $2-3 \mathrm{GHz}$ as below this the target polarimetric response is dominated by simple dipole moments. To counter this, the clutter polarization properties are very important. At low frequencies the clutter presents low entropy and hence more copolar variability, while at high frequencies the clutter response occupies more polarization space. This feature may explain why this method does not detect the mines at high frequencies.
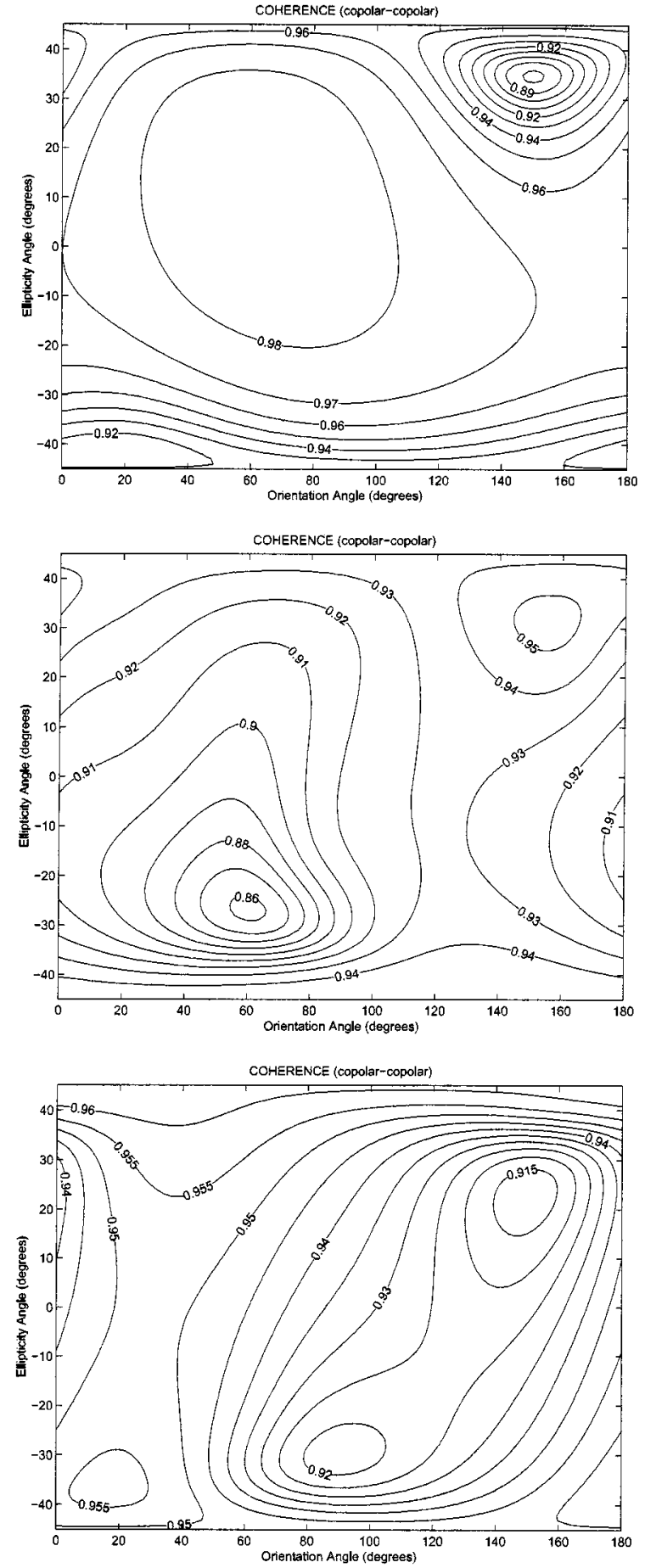

Fig. 7. Coherence as a function of polarization (copolar combinations) for three spatial positions: gravel (top), cylinder (center), and mine 3 (bottom). Frequency $=4-5 \mathrm{GHz} . B=0.5^{\circ}$.

From the previous discussions, we can also conclude that the polarimetric method presented here suffers from a strong dependency on the operating frequency. This dependency can be studied by representing a cut of the obtained height difference along the ground-range axis where mine 3 and mine 4 were buried, for different frequency ranges (see Fig. 9). The obtained height values are higher than expected due to no multi-look procedure being applied (in this plot) in order to preserve the spatial resolution and extract the correct position of the mines. Note 


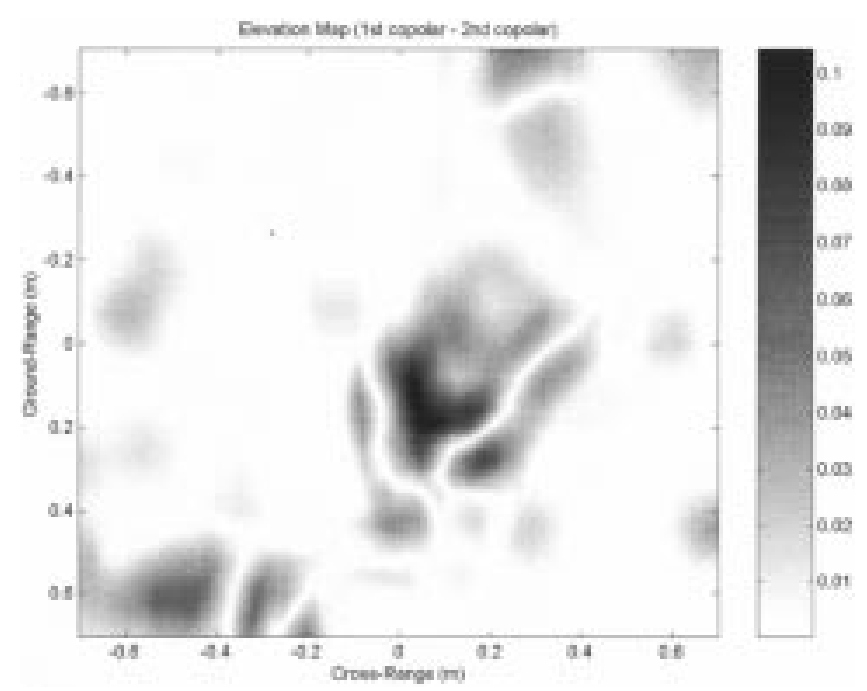

Fig. 8. Map derived from the height difference between the two optimum copolar polarizations at $4-5 \mathrm{GHz} . B=0.5^{\circ}$.

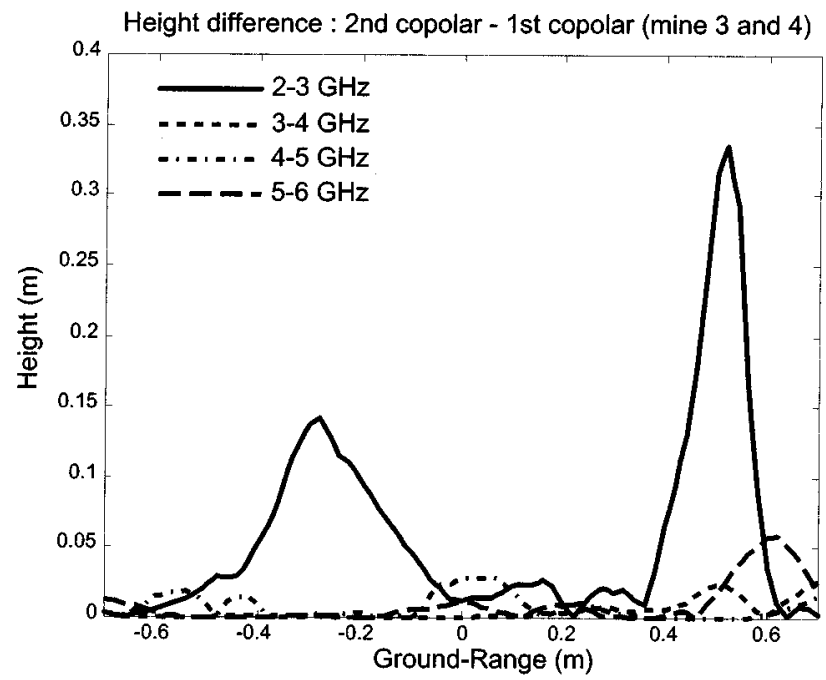

Fig. 9. Height difference between scattering centers at $x=0.4 \mathrm{~m}$ for several frequency bands. $B=0.5^{\circ}$.

that both mines are only detected with the lowest frequency band (2-3 GHz).

A final important issue in every interferometric technique is the effect of the baseline. Fig. 10 presents a comparison of the height differences derived by using three baselines: $0.25,0.5$, and $1^{\circ}$. As expected, with the smallest baseline the height estimation is less accurate due to phase noise, and some additional height peaks are observed. As a result, the false alarm probability for this baseline could be excessive. This is not the case at 0.5 and $1^{\circ}$. The results in Fig. 10 suggest that a multibaseline procedure might be suitable for the problem we are treating. A multibaseline technique would exploit, simultaneously, all interferometric information (coherences and phases) derived with different baselines.

\section{CONCLUSIONS}

In this paper we have presented a new polarimetric and interferometric approach that can be applied to improve the sub-

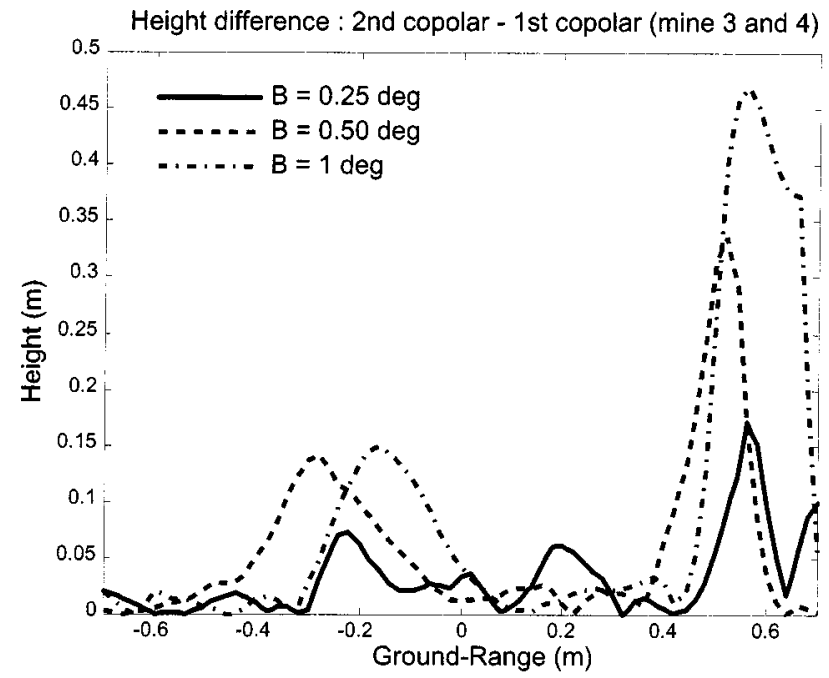

Fig. 10. Height difference between scattering centers at $x=0.4 \mathrm{~m}$ for several baselines. Frequency $=2-3 \mathrm{GHz}$.

clutter visibility in de-mining applications. The obtained experimental results have shown that buried plastic mines can not be detected by using only SAR images. However, after applying the method presented here, it is possible to separate the scattering mechanism that is associated with the clutter surface from that corresponding to the mines and estimate their height location. Nevertheless, the success of the proposed method will depend on the geometry of the problem. Indeed, the surface clutter and the mine must be physically apart in order to distinguish both scattering mechanisms when optimizing the interferometric coherence.

It has also been proved that the probability of detection shows a strong dependency on the frequency range. In case of detecting plastic mines, we have seen that it is convenient to operate at low frequencies in order to reduce the attenuation caused by ground propagation. On the other hand, multi-look averaging techniques must be applied in order to reduce the false alarm rate and improve the height estimation accuracy, at the expense of losing spatial resolution.

For the immediate future, a multibaseline approach appears as a logical refinement of this detection technique. Moreover, the minimum signal-to-clutter ratio needed for the method to successfully detect a buried target is still being studied. Related to this topic, much more analysis is required before making any final conclusion. In particular, the contribution of the global optimization method (theoretically better than the PSM) should be tested.

In this paper. we have not focused our interest on the discrimination between different buried targets (such as mines and stones). In principle, this discrimination could be approachable in two different ways. The first consists in the exploitation of the polarization states employed in the proposed procedure, because the coherence functions should be different for different targets. The second method is based on a sub-band processing. We have demonstrated that the results are different for different frequency bands, so all results could be combined to form a unified scheme. 
Finally, regarding the final application of this technique in real scenarios, some insight has been already obtained. The radar system should be fully polarimetric and operating at low microwave frequencies (roughly from 1 to $3 \mathrm{GHz}$ ). If possible, antennas should be deployed in a vertical configuration to form a simultaneous multibaseline system. Anyway, an extended set of experiments and a complete simulation analysis have to be performed to fully demonstrate the capabilities of this technique.

\section{ACKNOWLEDGMENT}

The authors would like to thank the personnel of the European Microwave Signature Laboratory for their support in performing the measurements and providing the experimental data. The authors would also like to thank the reviewers for their very helpful comments and discussions on the interpretation of the experiments.

\section{REFERENCES}

[1] L. Carin, N. Geng, M. McClure, J. Sichina, and L. Nguyen, "Ultrawide-band synthetic-aperture radar for mine-field detection," IEEE Antennas Propagat. Mag., vol. 41, pp. 18-33, Feb. 1999.

[2] S. R. Cloude and K. P. Papathanassiou, "Polarimetric SAR interferometry," IEEE Trans. Geosci. Remote Sensing, vol. 36, pp. 1551-1565, Sept. 1998.

[3] — "Polarimetric radar interferometry and its applications," in Proc. PIERS-Workshop on Advances in Radar Methods, Baveno, Italy, July 1998, pp. 61-63.

[4] A. J. Sieber, "The European Microwave Signature Laboratory," EARSel Adv. Remote Sensing, vol. 2, pp. 195-204, Jan. 1993.

[5] L. Sagues, J. M. Lopez-Sanchez, J. Fortuny, X. Fabregas, A. Broquetas, and A. J. Sieber, "Indoor experiments on polarimetric SAR interferometry," IEEE Trans. Geosci. Remote Sensing, vol. 38, pp. 671-684, Mar. 2000.

[6] H. A. Zebker and J. Villasenor, "Decorrelation in interferometric radar echoes," IEEE Trans. Geosci. Remote Sensing, vol. 30, pp. 950-959, Sept. 1992.

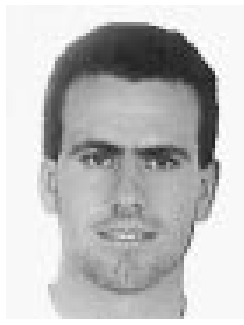

Lluís Sagués (SM'98) was born in Castellterçol, Barcelona, Spain, in 1972. He received the Ingeniero degree in telecommunication engineering from the Polytechnic University of Catalonia (UPC), Barcelona, in 1996, where he is currently pursuing the $\mathrm{Ph} . \mathrm{D}$. degree.

Since 1997, he has been with the Electromagnetics and Photonics Engineering Group of the Department of Signal Theory and Communications of the UPC working on radar polarimetry and SAR interferometry. His research interests include radar imaging, physical parameters retrieval of vegetated land surfaces, and buried object detection.

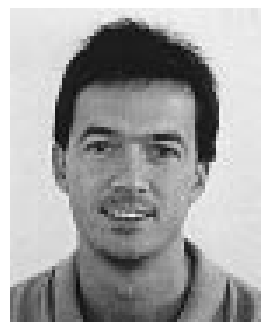

Juan Manuel Lopez-Sanchez (S'94-M'01) was born in Alicante, Spain, in 1972. He received the Ingeniero and Doctor Ingeniero degrees in telecommunications engineering from the Polytechnic University of Valencia (UPV), Valencia, Spain, in 1996 and 2000, respectively.

From 1998 to 1999 , he was a predoctoral grantholder at the Space Applications Insitute, Joint Research Centre of the European Commission, Ispra, Italy. In 2000, he joined the Departamento de Física, Ingeniería de Sistemas y Teoría de la Señal (DFISTS), University of Alicante, where he occupies a teaching position. His main research interests include analytical and numerical models for multiple scattering problems, microwave remote sensing for inversion of biophysical parameters, polarimetric techniques, and SAR imaging algorithms.

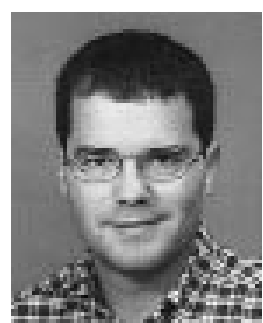

Joaquim Fortuny (S'93-A'96) was born in Tarragona, Spain, in 1964. He received the Ingeniero degree in telecommunications engineering from the Polytechnic University of Catalonia (UPC), Barcelona, Spain, in 1988.

From 1988 to 1989 , he worked on the design of microwave circuits at Ka-Band in the Antennas, Microwave, and Radar Group at the UPC. From 1990 to 1992, he was a Research Assistant in the RF Division at the European Space Technology Centre of ESA, The Netherlands. Since 1993, he has been with the Joint Research Centre of the European Commission, Ispra, Italy. His research interests are in radar imaging, subsurface sensing, and numerical techniques in electromagnetics.

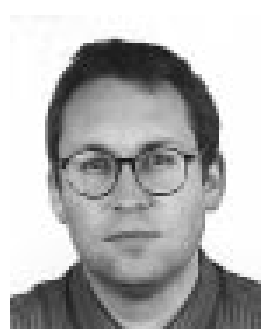

Xavier Fabregas (S'89-M'93) received the B.S. degree in physics from the Barcelona University, Barcelona, Spain, in 1988, and the Ph.D. degree in applied sciences from the Polytechnic University of Catalonia (UPC), Barcelona, in 1995.

Since 1996, he has been Associate Professor at the UPC. His current research interests include polarimetric retrieval algorithms, polarimetric calibration, and polarimetric SAR classification.

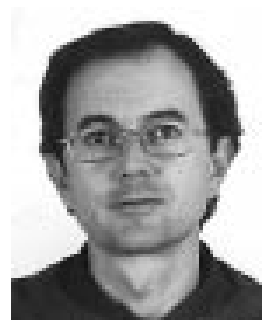

Antoni Broquetas (S'87-M'89) was born in Barcelona, Spain, in 1959. He received the Ingeniero degree in telecommunication engineering and the Doctor Ingeniero degree in telecommunications engineering, for his work on microwave tomography, from the Polytechnic University of Catalonia (UPC), Barcelona, Spain, in 1985 and 1989, respectively.

In 1986, he was a Research Assistant at the Portsmouth Polytechnic, U.K., involved in propagation studies. In 1987, he joined the Department of Signal Theory and Communications of the School of Telecommunication Engineering of the UPC. Currently, he is Professor of the UPC involved in research on radar imaging and remote sensing and responsible of the research activities of the recently created Institute of Geomatics.

Alois J. Sieber (M'80-SM'86-F'00) received the M.S. degree in physics from the Technical University of Karslruhe, Karslruhe, Germany, in 1971, the Ph.D. degree in 1973 from the University of Tübingen, Germany, and the Habilitation degree in remote sensing in 1985 from the University of Stuttgart, Germany.

Since 1986, he has been with the Joint Research Centre of the European Commission, Ispra, Italy. He is the head of the unit Technologies for Detection and Positioning, Space Applications Institute. Among other activities, he is also the head of the European Microwave Signature Laboratory (EMSL). Since 1986, he has been a Senior Lecturer at the University of Stuttgart. His research interests are in remote sensing, radar imaging, subsurface sensing, and numerical techniques in electromagnetics. He is leading several projects supporting the goal to overcome the global scourge of landmines within the foreseeable future. 